\title{
ON SOME CONJECTURES REGARDING TRIDIAGONAL MATRICES
}

\author{
Carlos M. da Fonseca
}

Kuwait College of Science and Technology, Doha District, Block 4, P.O. Box 27235, Safat 13133

Kuwait

University of Primorska, FAMNIT, Glagoljsaška 8

6000 Koper, Slovenia

c.dafonseca@kcst.edu.kw, carlos.dafonseca@famnit.upr.si

Received: 18 December 2018; Accepted: 20 February 2019

\begin{abstract}
We discuss several conjectures proposed recently by A.Z. Küçük and M. Düz on the permanent of certain type of tridiagonal matrices. We recall some less known results on tridiagonal matrices and, at the same time, bring other results together to a common framework.
\end{abstract}

MSC 2010: 15A18,15B05

Keywords: tridiagonal matrices, determinant, permanent, Chebyshev polynomials of second kin

\section{Introduction}

László Losonczi was perhaps the first to study, in [1,2], the matrices of type

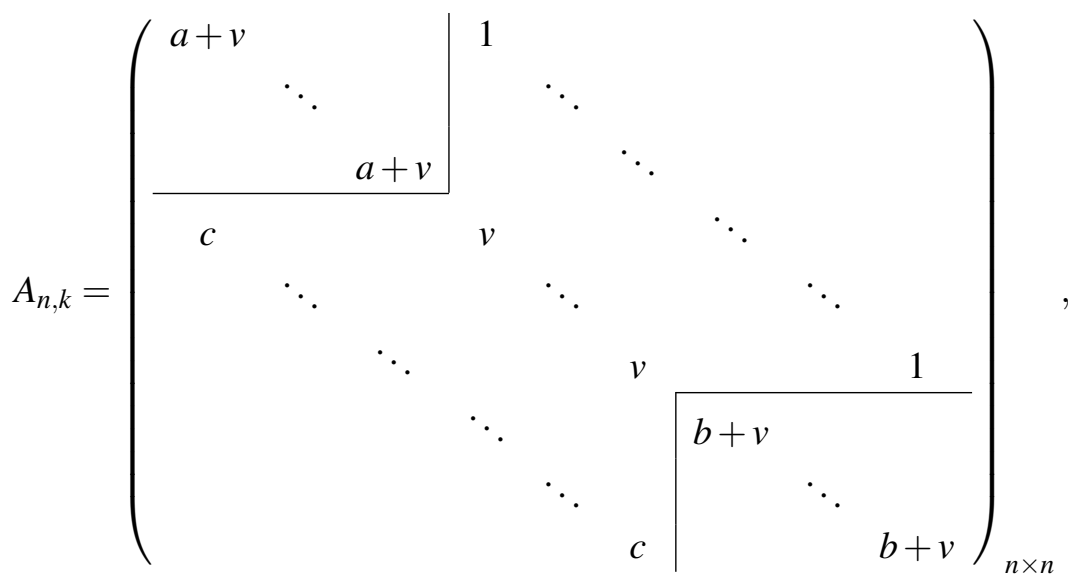

with $k \times k$ upper left and bottom right principal submatrices and $n-2 k \geqslant 0$, where the non-mentioned entries are to be read as zero. Namely, we can find 
Theorem 1 ( [1]) Let $n=q k+r$, with $0 \leqslant r<k$. Then

$$
\operatorname{det} A_{n, k}=\left(\operatorname{det} A_{q+1,1}\right)^{r}\left(\operatorname{det} A_{q, 1}\right)^{k-r} .
$$

For $n-2 k<0$, the definition is slightly different and it is not a focus of our attention here.

The band matrices $A_{n, k}$ are a special case of $k$-tridiagonal matrices. In fact, when $a+v=b+v=v$, they are known as $k$-tridiagonal Toeplitz matrices. For an interpretation of (1) in terms of graph theory, the reader is referred to [3]. The case when the upper and bottom blocks are not square was analysed in [4]. However, one should differentiate this notion from the tridiagonal $k$-Toeplitz matrices, where the diagonals follow a certain periodic pattern [5].

Naturally we have, in particular,

$$
A_{n, 1}=\left(\begin{array}{cccccc}
a+v & 1 & & & & \\
c & v & 1 & & & \\
& c & \ddots & \ddots & & \\
& & \ddots & \ddots & 1 & \\
& & & c & v & 1 \\
& & & & c & b+v
\end{array}\right)_{n \times n},
$$

which is a monic Jacobi matrix. For more general perturbations the reader is referred to [6].

From [1, Theorem 2 (7)], we have

$$
\operatorname{det} A_{n, 1}=(\sqrt{c})^{n}\left(U_{n}\left(\frac{v}{2 \sqrt{c}}\right)-\frac{a+b}{\sqrt{c}} U_{n-1}\left(\frac{v}{2 \sqrt{c}}\right)+\frac{a b}{c} U_{n-2}\left(\frac{v}{2 \sqrt{c}}\right)\right),
$$

where $\left\{U_{n}\right\}_{n \geqslant 0}$ are the Chebyshev polynomials of second kind of degree $n$. We recall that these polynomials satisfy the three-term recurrence relations

$$
2 x U_{n}(x)=U_{n+1}(x)+U_{n-1}(x), \quad \text { for all } n=0,1,2, \ldots,
$$

with initial conditions $U_{-1}(x)=0$ and $U_{0}(x)=1$, and an explicit formula for $U_{n}$ is

$$
U_{n}(x)=\frac{\sin (n+1) \theta}{\sin \theta}, \quad \text { with } x=\cos \theta \quad(0 \leqslant \theta<\pi) .
$$

These results and their consequences have largely been ignored in the literature, rediscovered, and replicated by many authors for the past decades since Losonczi established them as one can attest in [7,8]. For instance, from (1) and (2), one can find the eigenvalues of many perturbed Toeplitz tridiagonal matrices.

Recently A.Z. Küçük and M. Düz proposed in [9] several conjectures on the permanent of a certain family of $A_{n, k}$. In this note, our aim is to prove two conjectures using Losonczi's results relating the permanent and the determinant of two families 
of $k$-tridiagonal Toeplitz matrices. We will reshape the main results of [9] in terms of the existing literature.

\section{The known results}

In [9], Küçük and Düz considered the matrix

$$
T_{n}^{(k)}=\left(\begin{array}{ccc|cccccc}
2 x & & & & & & & & \\
& \ddots & & & \ddots & & & & \\
& & 2 x & & & \ddots & & & \\
\cline { 1 - 4 } & & & & & & \ddots & & \\
& \ddots & & & \ddots & & & \ddots & \\
& & \ddots & & & 2 x & & & i \\
& & & \ddots & & & 2 x & & \\
& & & & \ddots & & & \ddots & \\
& & & & & i & & & 2 x
\end{array}\right)_{n \times n},
$$

and proposed several conjectures for its permanent. Recall that the permanent of a square matrix equals the sum of the weights of all cycle-covers of its underlying directed graph. Since the undirected graph of $T_{n}^{(k)}$ is always acyclic, i.e., cycle-free, the permanent of $T_{n}^{(k)}$ is the permanent of the matrix

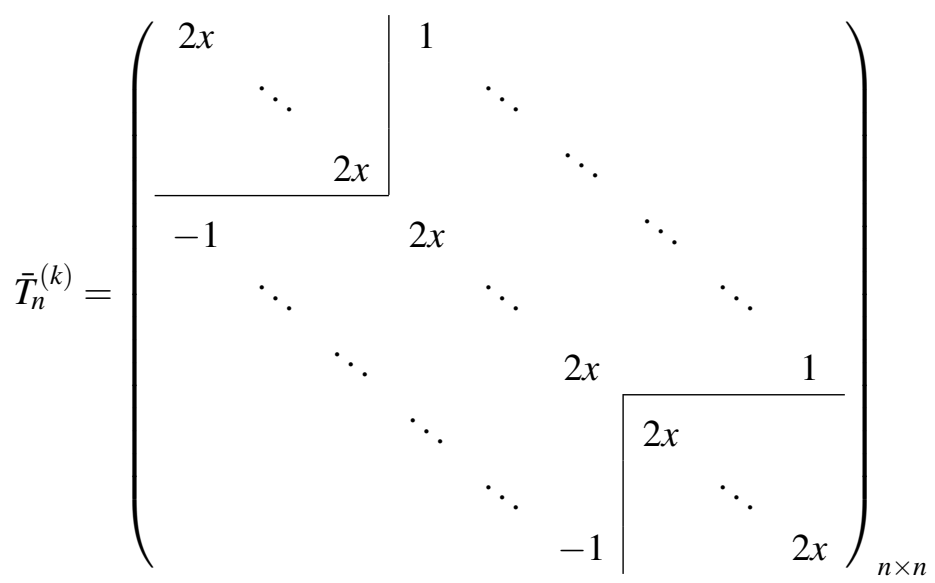

The matrix $\bar{T}_{n}^{(k)}$ is convertible (cf. [10]) in the sense that changing the signs of the subdiagonal of -1 's we get a matrix whose determinant equals the permanent of $\bar{T}_{n}^{(k)}$, i.e.,

$$
\operatorname{per} T_{n}^{(k)}=\operatorname{det} \tilde{A}_{n, k}
$$


where the new matrix, say $\tilde{A}_{n, k}$, is $A_{n, k}$ with $a=b=0, c=1$, and $v=2 x$. This means, for example, that the permanent of $T_{n}^{(1)}$ is

$$
\operatorname{det} \tilde{A}_{n, 1}=U_{n}(x),
$$

which is precisely Theorem 1 in [9]. More generally, we have

$$
\operatorname{det} \tilde{A}_{n, 1}=U_{k}(x) U_{\ell}(x)-U_{k-1}(x) U_{\ell-1}(x),
$$

when $k+\ell=n$.

A sum decomposition for $\operatorname{det} \tilde{A}_{n, 2}$ is also known. From (1) we have

$$
\operatorname{det} \tilde{A}_{n, 2}=U_{q+1}^{r}(x) U_{q}^{2-r}(x),
$$

where $n=2 q+r$, with $0 \leqslant r<2$. Then, depending on the parity of $n$, we combine 22.12.4 or 22.12 .5 with 22.7 .25 in [11] to readily obtain

$$
\operatorname{det} \tilde{A}_{n, 2}=\sum_{\ell=0}^{q} U_{n-2 \ell}(x) .
$$

This result is [9, Theorem 2]. However, the proof presented in [9] is long and inaccurate.

Of course, from (6), $\operatorname{det} \tilde{A}_{n-2,2}=U_{q}^{r}(x) U_{q-1}^{2-r}(x)$. Therefore, one gets for $n>2$,

$$
\operatorname{det} \tilde{A}_{n, 2}-\operatorname{det} \tilde{A}_{n-2,2}=\left\{\begin{array}{cc}
U_{q}^{2}(x)-U_{q-1}^{2}(x), & \text { if } n \text { is even } \\
U_{q+1}(x) U_{q}(x)-U_{q}(x) U_{q-1}(x), & \text { if } n \text { is odd. }
\end{array}\right.
$$

In any case, from (5) and (4), we always get $U_{n}(x)$. This is the result one can find in [9, Theorem 3].

\section{The conjectures}

It results from the discussion above, that [9, Conjecture 4] cannot be posed. In fact, one should look at (1), (6), and the remainder of the division $n$ by 3 . However, the aforementioned conjecture is posed in terms of the parity of the matrix orders. On the other hand, the imposition of the condition (n symbol 6) is useless.

The next conjecture is [9, Conjecture 8].

Conjecture 1 If $n \equiv 1(\bmod k)$, with $n \geqslant k+1$, then

$$
\operatorname{det} \tilde{A}_{n, k}=2 x \operatorname{det} \tilde{A}_{n-1, k}-\operatorname{det} \tilde{A}_{n-2, k} \text {. }
$$

This conjecture is true and it can be proved as follows. Let us assume that $n=q k+1$. Then $n-1=q k$ and $n-2=(q-1) k+k-1$. Thus we have successively 


$$
\begin{aligned}
\operatorname{det} \tilde{A}_{n, k} & =U_{q+1}(x) U_{q}^{k-1}(x) \\
& =\left(2 x U_{q}(x)-U_{q-1}(x)\right) U_{q}^{k-1}(x) \\
& =2 x U_{q}^{k}(x)-U_{q}^{k-1}(x) U_{q-1}(x) \\
& =2 x \operatorname{det} \tilde{A}_{n-1, k}-\operatorname{det} \tilde{A}_{n-2, k} .
\end{aligned}
$$

We observe that with this proof, [9, Conjecture 10] is exactly (8) and thus attest its veracity. Finally we remark that $[9$, Theorem 7] is the particular case (7) when $k=2$.

\section{Conclusions}

In this note, we discussed the conjectures proposed recently by A.Z. Küçük and M. Düz in [9]. We proved positively some of them. The main notions were recapitulated in terms of the existing literature, namely the paper [1].

\section{References}

[1] Losonczi, L. (1992). Eigenvalues and eigenvectors of some tridiagonal matrices. Acta Mathematica Hungarica, 60, 309-332.

[2] Losonczi, L. (1987). On some discrete quadratic inequalities. International Series of Numerical Mathematics, 80, 73-85.

[3] da Fonseca, C.M., \& Yilmaz, F. (2015). Some comments on $k$-tridiagonal matrices: determinant, spectra, and inversion. Applied Mathematics and Computation, 270, 644-647.

[4] McMillen, T. (2009). On the eigenvalues of double band matrices. Linear Algebra and its Applications, 431, 1890-1897.

[5] da Fonseca, C.M., \& Petronilho, J. (2005). Explicit inverse of a tridiagonal $k$-Toeplitz matrix. Numerische Mathematik, 100(3), 457-482.

[6] da Fonseca, C.M., Kouachi, S., Mazilu, D.A., \& Mazilu I. (2015). A multi-temperature kinetic Ising model and the eigenvalues of some perturbed Jacobi matrices. Applied Mathematics and Computation, 259, 205-211.

[7] Borowska, J., \& Łacińska, L. (2015). Eigenvalues of 2-tridiagonal Toeplitz matrix. Journal of Applied Mathematics and Computational Mechanics, 14, 11-17.

[8] Borowska, J., Łacińska, L., \& Rychlewska, J. (2013). On determinant of certain pentadiagonal matrix. Journal of Applied Mathematics and Computational Mechanics, 12, 21-26.

[9] Küçük, A.Z., \& Düz, M. (2017). Relationships between the permanents of a certain type of $k$-tridiagonal symmetric Toeplitz matrix and the Chebyshev polynomials. Journal of Applied Mathematics and Computational Mechanics, 16, 75-86.

[10] da Fonseca, C.M. (2011). An identity between the determinant and the permanent of Hessenberg type-matrices. Czechoslovak Mathematical Journal, 61(136), 917-921.

[11] Abramowitz, M., \& Stegun, I.A. (1972). Handbook of Mathematical Functions with Formulas, Graphs, and Mathematical Tables, Tenth printing. National Bureau of Standards, Applied Mathematics Series 55. 